Physical Sciences | Prof Martín Jaraíz

\title{
Quantum molecular modelling for efficient chemical synthesis
}

Computer Aided Design informs the world around us, but it has taken a long time to have a significant impact on the chemical synthesis industry. Professor Martin Jaraiz at the University of Valladolid in Spain has proposed a novel approach tool called Density Functional Theory (DFT) in combination with microkinetic modelling to more accurately predict the effect of catalysts and additives on reaction performance. The proposed DFT-based modelling protocol is more computationally efficient and has the same or higher accuracy compared he modern world is full of things
designed on computers: your car, computer iself. Computer Aided Design (CAD) is an essential tool for creating, modifying and optimising designs in and electronic engineering. But what about the chemical engineers creating pharmaceuticals, cosmetics and other sy hadic chemicals - can they utilise question is "yes".

The big difference between designing buildings and chemical reaction mechanisms is the quantum mechanics involved in modelling molecules: the bricks and mortar of the chemical world are atoms with slightly blurry edges, and electrons with probability densities rather than definite locations.

\section{MICROKINETIC MODELLING} Modelling chemicals and their interactions - known as microkinetic modelling - is tricky, but there are in fact many methods. One of the most successful, Density Functional Theody (DF), is a utilisation of quantum mechanics to investigate the movement technology is still developing, and recent progression in DFT software allows synthetic chemists to make complex predictions of how molecules interact. than ever before, allowing chemists to choose effective catalysts and predict the effect of additives on a reaction.

Synthetic chemists are interested in producing new molecules and improving $\mathrm{c}$ production methods. These reactions, Figure $1 \mathrm{~A}$, often inclu multiple starting materials and products, and a successful compun Over the course of a reaction, Figure 1B, ( which also need to be accuned for If that's not enough, catalysts - substances which don't get used up in the reaction re regenerated, creating cycles, Figure D. Taking all of these into account form large network or 'eaction scheme' by reversible or irreversible reactions.

Typically, researchers using DFT methods start with a suspected full reaction scheme, which may contain
more than 100 individual reactions. The energetics of each reaction within the scheme determine how easy it is for that reaction step to proceed. In the standard protocol, the majority of energetic parameters are fixed at the DFT calculated value, leaving a limited number of parameters that can be varied within the error boundaries of the calculation until the simulation matches experimental values. This process can be time-consuming, due to the a large number of DFT parameters.

\section{TIGHTENING UP THE PROCEDURE} Over the last three years, Professor Mart Jaraíz at the University of Valladolid in Spain has been using another method one which provides not only equally or but also requires less experimental input and computation time. Rather than starting with every possible reaction pathway, instead begin with just the dominant mechanisms - Jaraiz calls these 'loose' and 'tight' modelling approaches. Starting with less information may seem counter-intuitive, but because fewer pactions are being modeled, all the parameters can be adjusted in the DFT pre in place, imponontationis
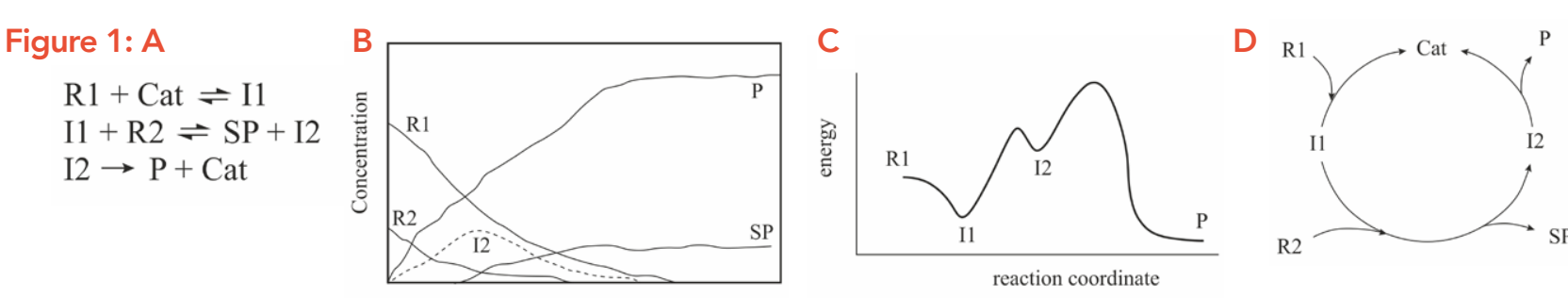
A reaction system (A) determines the
pathways $(D)$ of the reaction system.

reactions can be added to fine-tune the model based on experimental data.

Building a model made up of many individual chemical reactions requires specific methods and tools of its ow In the laboratory, Reaction Progress Kinetic Analysis (RPKA) can be used to identify rate laws of the dominant mechanisms - that is, how quickly chemicals. Outside the lab, theretica proaches like Automated Reaction Path Searches can elucidate the important pathways and transition states. In combination, these can be used to build a reaction scheme of the various reactions that eventually lead to the synthesis of a molecule. The tight method requires initially identifying a minimum number of reactions that can explain the overall reaction.

Once a basic reaction scheme has been proposed, the next step of the DFT process is to consider more deep

the energetics of each reaction step If all the reactions in a mechanism are energetically plausible, then the On the other hand, models containing

valuable for explaining experiment spectroscopic observations, such as the slow build-up of particular

Recent progression in the combined use of DFT and microkinetic software allows synthetic chemists to predict the outcome of complex reaction systems.

reactions which are energetically It is also important to consider the fect of fluctuating levels of on reversible reactions and their transition states.

Once each reaction in the initial model has earned its place in the

scheme, additional information can be incrementally added to the model for fine-tuning. These details are

\section{VERIFYING A PROPOSED MOD} this new protocol to a series of case involving titanocene monochloride $\left(\mathrm{Cp}_{2} \mathrm{TiCl}\right.$, a catalyst sometimes used in ring-opening reactions of epoxides. A mild acid called collidine hydrochloride (CollHCl) is often added to this reaction, which prevents the

$\mathrm{C}_{2} \mathrm{TiCl}$ from being deactivated over the course of the reaction. The resulting and converts
Figure 2

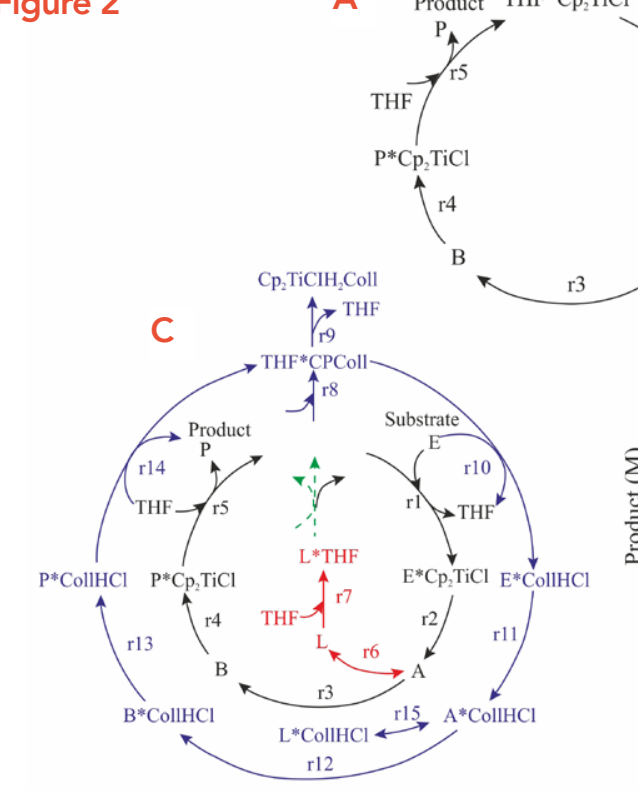

Example of model development following the incremental tight modelling protocoll Experimental data (symbols) and simulation (lines) for two sets of 
almost all of the substrate into the cired product, with only a small Catalyst deactivation is a very important factor when deciding upon catalytic processes for large-scale industria chemical synthesis.

Jaraíz and his team began their DFT modelling process with a reaction scheme (Fig. 2A) of five key reaction making up the catalytic cycle. The initial scheme did not take into account catalyst deactivation and the crucial role of CollHCl to suppress deactivation, in order to begin with a simpler reaction system for DFT. Only once the energetics of these reaction were established (i.e. calculated and fine-tuned), Jaraiz modified the reaction for deactivation (Fig 2B) and the effect of the CollHCl additive (Fig. 2C).

To test the predictive capability of the procedure, the model was trained on only three experimental data points (Fig. 2A), and the data was collected from a reaction performed without CollHC results from the synthesis done with

Figure 3

\begin{tabular}{|c|c|}
\hline $\begin{array}{l}\text { Loose } \\
\text { (commonly used) }\end{array}$ & $\begin{array}{c}\text { Tight } \\
\text { (proposed) }\end{array}$ \\
\hline $\begin{array}{l}130 \text { reactions } \\
\text { (All plausible mechanisms) }\end{array}$ & $\begin{array}{l}8 \text { reactions } \\
\text { (Only dominant mechanisms) }\end{array}$ \\
\hline $\begin{array}{l}149 \text { DFT parameters } \\
\text { (Only } 9 \text { adjusted) }\end{array}$ & $\begin{array}{l}14 \text { DFT parameters } \\
\text { (All adjusted) }\end{array}$ \\
\hline $\begin{array}{l}X \text { Can lead to wrong } \\
\text { conclusions }\end{array}$ & $\begin{array}{l}\checkmark \text { Same or better accuracy } \\
\checkmark>10 \text {-fold DFT time savings } \\
\checkmark \text { Incremental modelling }\end{array}$ \\
\hline
\end{tabular}

The commonly used modelling approach (loose) includes many DFT parameters but, due to the limited experimental latat, can only fine-tune a few of them. The proposed approach (tight) starts with only ythe
main, dominant mechanisms (reactionss), adjusts all the DFT parameters, and proceeds incrementally .

$\begin{array}{ll}\text { from a recently published reaction } & \text { was fine-tuned using only data from five } \\ \text { scheme, proposed by another research } & \text { experiments. By this point the simulation }\end{array}$ group: the ring opening reaction of 1,2 epoxyoctane by 2 propanol, catalysed by fluorinated aryl borane (FAB).

This reaction is interesting because the presence of unwanted water can cause the regioselectivity of $F A B$ to decrease,

The use of predictive, DFT-based reaction simulators could have a significant economic impact on the chemical industry and without CollHCl. The model was very successful. It could very accurately $A, B$ ) and several other reagents.

For another case study, even the ascalculated DFT parameters predicted correct yields, the only difference with eaction to occur about 360 times faster than it actually does. After fine-tuning the DFT parameters, the simulator could match the timescale for the reaction

as well.

\section{PROPOSING A RELIABLE}

MODELLING PROTOCOL

Another case study illustrates the

potency of the "light method and how

3). For this case study Jo esults (Fig.

3). For this case study, Jaraiz chose as resulting in lower amounts of the desired product. A reaction scheme had been previously proposed by another research group, created using the often used odelling approach. To explain the effect of water on the reaction, the scheme contained 130 reactions, resulting in 149 DFT parameters. Given the large number of parameters, only nine were adjusted, and the remainder were set at the calculated DFT values.

For a tight modelling approach, Jaraíz chose eight for the initial scheme of 130. This resulted in a reduction in the number of DFT parameters to 14 , all of which could be adjusted. This initial reaction scheme included the catalytic cycle of $F A B$ to convert the substrate in the product, two deactivation reactions creating side-products. The simulo
Was fine-tuned using only data from five could predict the relationship between the amount of water and both the initial with high accuracy.

The biggest discrepancy between the tight and loose models is in the relationship between water level and regioselectivity. The loose model than is actually seen in experiments, whereas the tight model provides more accurate values and did not include model deent these stepsunhe tight and, thus, reduces the chances of for example, predicting multiple catalytically relevant species and multiple catalytic pathways whose existence is questionable and that result from the large number of reactions that have only been loosely adjusted and tested.

\section{CHEMICAL CAD}

In summary, the tight protocol proposed by Prof Jaraíz is a highly effective substitute for the previous protocol that can provide more robust and reliable models and also drastically decreases computation time. CAD can increasingly be used for chemical synthesis, and this nove mplementation or DrF based kinetic predictive CAD for chemical processes. rate and regioselectivity of the reaction predicted a much stronger relationship some unconventional water mediated

\section{Behind the Research}

Prof Martín Jaraíz

E: mjaraiz@ele.uva.es T: +34983423677 W: https://www.researchgate.net/profile/M_Jaraiz

\section{Research Objectives}

Professor Jaraiz's research is focused on the 作 or materials processing.

\section{Detail}

Department of Electronics, ETSIT, University of Valladolid, Paseo de Belen 15, Valladolid 47011, Spain

Professor Martín Jaraiz obtained his Ph.D. from the University of Valladolid in 1981. He has carried out kinetic Bell Laboratories, New Jersey and Synopsys Inc., California. While exploring molecular electronics he became interested on improving reaction kinetics modelling for synthetic chemistry in general.

Funding

MINECO-FEDER (CTQ2015-68148-C2) and Junta de Castilla y León (VA056G18)

\section{Collaborators}

DrJose E. Rubio (Univ. Valladolid) - DrLourdes Enriquez (Univ. Valladolo - Prof José L López Péra (Unide - Prof Alberto Lesarri (Univ Valladenama
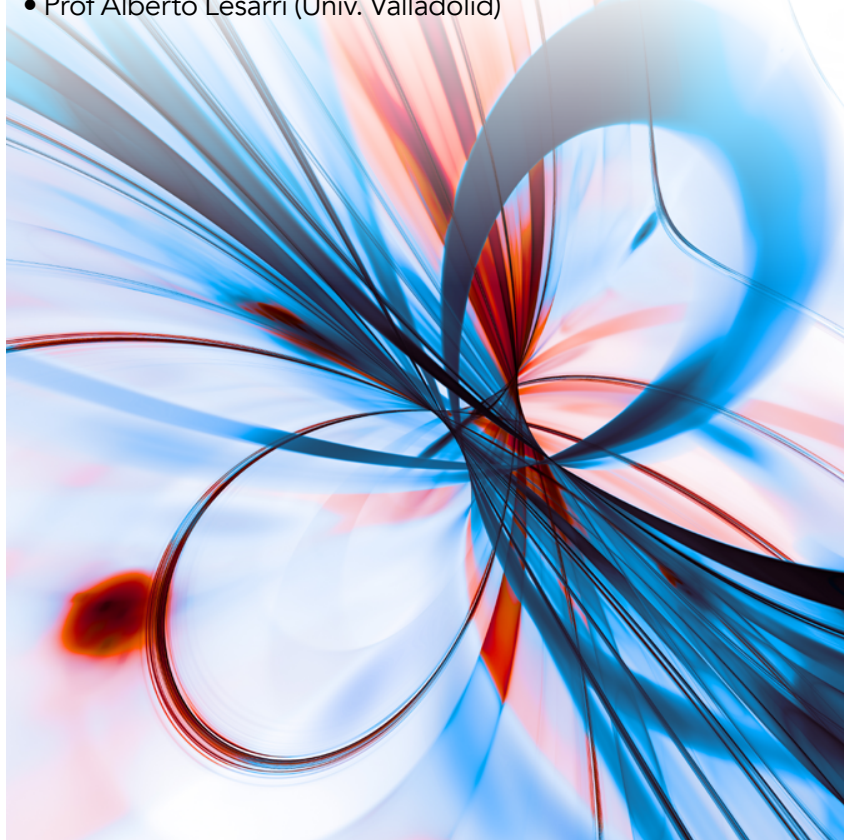

\section{References} Jaraíz, M; Rubio JE; Enríquez, L; Pinacho, R; Lopez-Pérez, Protocol: Start with Only the Dominant Mechanisms, Adjust All Parameters, and Build the Complete Mode Incrementally. ACS Catalysis, 9, 4804-4809. https://doi. Jaraíz, M; Enríquez, L; Pinacho, R; Rubio JE; Lesarri, A;
Lopez-Péreź, JL. (2017). A DFT-Based ComputationalExperimental Methodology for Synthetic Chemistry: Example of Application to the Catalytic Opening of Epoxides by Iitanocene. Journal of Organic Chemistry,

\section{Personal Response}

To what extent do industry use predictive techniques from more implementation of these methods?

II Kinetic simulations have been used for decades,

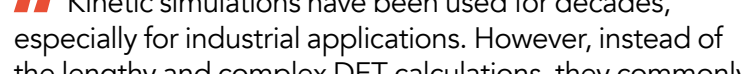
the lengthy and complex DFT calculations, they commonly expertise, and the parameters are adjusted from dedicated experiments. We can expect that the widespread use of predictive computational tools such as DFT-based plannedicteraction between computation and axperiellwill contribute to a synthetic chemistry less based on trial and error and more on predictive assessments. In addition considering the yearly volume of industrial chemical

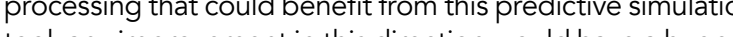
. 